\title{
Biomedical signals and machine learning in amyotrophic lateral sclerosis: a systematic review
}

\author{
Felipe Fernandes ${ }^{1^{*}+}$ (D), Ingridy Barbalho ${ }^{1 \dagger}$, Daniele Barros ${ }^{1}$, Ricardo Valentim ${ }^{1}$, César Teixeira ${ }^{2}$, Jorge Henriques ${ }^{2}$, \\ Paulo Gil ${ }^{2}$ and Mário Dourado Júnior ${ }^{1}$
}

\author{
*Correspondence: \\ felipe.ricardo@lais.huol. \\ ufrn.br \\ ${ }^{\dagger}$ Felipe Fernandes and \\ Ingridy Barbalho contributed \\ equally to this work \\ ${ }^{1}$ Laboratory of Technological \\ Innovation in Health (LAIS), \\ Federal University of Rio \\ Grande do Norte (UFRN), \\ Natal, RN, Brazil \\ Full list of author information \\ is available at the end of the \\ article
}

\begin{abstract}
Introduction: The use of machine learning (ML) techniques in healthcare encompasses an emerging concept that envisages vast contributions to the tackling of rare diseases. In this scenario, amyotrophic lateral sclerosis (ALS) involves complexities that are yet not demystified. In ALS, the biomedical signals present themselves as potential biomarkers that, when used in tandem with smart algorithms, can be useful to applications within the context of the disease.
\end{abstract}

Methods: This Systematic Literature Review (SLR) consists of searching for and investigating primary studies that use ML techniques and biomedical signals related to ALS. Following the definition and execution of the SLR protocol, 18 articles met the inclusion, exclusion, and quality assessment criteria, and answered the SLR research questions.

Discussions: Based on the results, we identified three classes of ML applications combined with biomedical signals in the context of ALS: diagnosis (72.22\%), communication (22.22\%), and survival prediction (5.56\%).

Conclusions: Distinct algorithmic models and biomedical signals have been reported and present promising approaches, regardless of their classes. In summary, this SLR provides an overview of the primary studies analyzed as well as directions for the construction and evolution of technology-based research within the scope of ALS.

Keywords: Amyotrophic lateral sclerosis_-ALS, Artificial intelligence, Biomedical signals, Chronic neurological conditions, Machine learning, Motor neuron disease, Signal processing

\section{Background}

Amyotrophic lateral sclerosis is a disease characterized by the progressive and irreversible degeneration of motor neurons, which causes deficits in the ability to control movement, breathing, and, in $50 \%$ of cases, in cognitive and behavioral functioning [1-3]. The cause of ALS is still unknown and there is no treatment to cure it. Hence, there are only alternatives of palliative care and medication to delay the progress of the disease $[4,5]$. 
Diagnosing patients with ALS represents a challenging task due to its complex pathogenesis and the absence of specific biomarkers $[6,7]$. The diagnosis is based on clinical presentation, progression of symptoms, and the exclusion of other diseases supported by tests such as Electromyography (EMG). Such a process requires an average of 10-18 months from the onset of symptoms to confirmation [8-11]. What is more, the diagnosis is considered slow and late given the characteristics of ALS, in which life expectancy after confirmation is of $2-5$ years [12].

Despite being described by Jean-Martin Charcot more than 100 years ago [13], ALS is considered a rare disease, and, to this date, there are not many countries with records of epidemiological data. In a few European countries, as well as in the United States, epidemiological records show that the incidence rate of ALS is of 1-2 cases per 100,000 individuals per year, while the prevalence is approximately 5 cases per 100,000 individuals, which for van Es et al. [3] reflects the fast lethality of the disease. A worldwide increase in the number of ALS-affected individuals is expected, rising from 222,801 cases in 2015 to 376,674 by 2040 , according to the projection made by Arthur et al. [14]. The aging of populations and the consequent rise in the number of individuals within the age group with a more considerable risk for ALS, which is of 60-79 years, represent the probable culprits for the $69 \%$ worldwide increase [14].

Considering the intrinsic aspects of ALS, it is critical to promptly search for diagnostic support systems, as well as for alternatives that intermediate essential communication, autonomy, and promote quality of life to patients. From this standpoint, several technology-based studies have been developed. These investigations typically provide auxiliary resources for diverse aspects regarding ALS, going from what pertains to patients and their caregivers to matters related to outpatient care in organizational health entities [15-17].

Technologies developed for health encompass and collaborate in positive progressions in remarkable ways, such as with the diagnosis of ALS [18, 19], monitoring of disease progression [20], monitoring of food intake [21], communication intermediation [22-25], autonomy [26], and other applications based in artificial intelligence, as it has been reviewed by Schwalbe and Wahl [27]. Automated systems for disease diagnosis, for instance, are computational tools composed of ML techniques that, based on the processing of biomedical signals, are capable of aiding the detection of neuromuscular disorders [28]. These systems contain expert information of specific domains, which provide health professionals with decision-making support and represent strategies and measures adopted in the care of patients [29].

Recently, in the context of ALS, Grollemund et al. [30] published a comprehensive review that presents and investigates ML models. Thus, it uses or combines different data types from individuals with ALS (clinical, genetic, biological clinical, and imaging), in three-class applications: diagnosis, prognosis, and risk stratification. In conclusion, the authors point to promising advances with this approach in the academic and clinical field in the ALS ecosystem. In this perspective, this SLR complements Grollemund et al. [30] in analyzing ML models in applications for ALS using specifically biomedical signals.

Biomedical signals consist of data from a studied physiological system and their processing aims mainly to extract relevant information [31,32]. This information can 
enhance data-driven artificial intelligence techniques, especially ML algorithms, and it is used to support the diagnosis of various diseases [27]. There are several types of biomedical signals, as EMG, electroencephalogram (EEG), electrocardiogram (ECG), electrooculogram (EOG), gait rhythm (GR) and magnetic resonance imaging (MRI). Regarding the ML models, Artificial Neural Network (ANN), decision tree (DT), support vector machine (SVM), and K-Nearest Neighbor (KNN) are particular examples of techniques that have been extensively considered in the healthcare realm, including in the context of ALS [33-36].

\section{Objective}

The chief goal of this systematic literature review is to investigate ML-based approaches, in tandem with the biomedical signals, that contribute to the practical and scientific advancement of aspects in the field of ALS. In this manner, it is expected to provide an overview of the matter at hand, considering the identification of the most-used biomedical signals and ML-based models, in addition to gathering details of primary studies, such as the purpose, the performance of algorithmic models, and experimental data, to identify strengths and opportunities for future researches.

\section{Methods}

We have developed this research considering the systematic review guidelines proposed by Kitchenham [37]. In the perspective of investigating technological applications in ALS, this study aims at (i) identifying the most applied biomedical signals; (ii) identifying for what purposes those are used; and (iii) verifying the usage of ML techniques or intelligent approaches to the processing of those signals. Hence, the research questions (RQ) were elaborated on this premise (see Table 1, presented below).

The primary studies searching and screening process in the scientific databases were categorized into four stages, according to what is displayed in Fig. 1. In the first stage, an initial set of articles was selected from the output of searches carried out in the IEEE Xplore, Web of Science, Science Direct, Springer, and PubMed databases. The following search strings (STR) were used in this first stage:

- STR01: ((("signals processing" OR "signals biomedical”) OR ("smart systems" OR "machine learning" OR "artificial intelligence" OR "computational intelligence" OR "algorithm" OR "algorithms")) AND ("amyotrophic lateral sclerosis" OR "als"));

Table 1 Research questions

\begin{tabular}{ll}
\hline RQ & Description \\
\hline 01 & For what purpose is the processing of signals used? \\
02 & What are the types of signals analyzed by the study? \\
03 & What intelligent techniques are used in the study? \\
04 & What is the performance of the analyzed techniques? \\
05 & How many subjects are used to test or validate the study?
\end{tabular}




\section{Identify and organize the studies returned from the databases. \\ 2 Apply inclusion criteria. \\ 3 Apply exclusion criteria. \\ 4. Analyze articles according to quality criteria.}

Fig. 1 Methodology steps

- STR02: ((("signals processing” OR "signals biomedical”) OR ("intelligent systems" OR "machine learning” OR "artificial intelligence" OR "algorithms" OR "Computational Intelligence")) AND ("amyotrophic lateral sclerosis" OR "als")).

In the second stage, the predefined inclusion criteria (IC), presented in Table 2, were applied to the initial set of articles from the previous phase. Primarily, an IC delimits the boundaries or scope of the investigation and possibilities the generation of a new subset of papers with a more significant probability of answers to the RQ. In such a context, the subset includes research articles from the last ten years that have been published in journals and are directly related to the principal area of interest of this systematic review.

Table 2 Inclusion criteria

\begin{tabular}{ll}
\hline IC & Description \\
\hline 01 & Articles published between 2009 and 2019 \\
02 & Research articles published in Journals \\
03 & $\begin{array}{c}\text { Articles in the areas of technology, engi- } \\
\text { neering or computer science }\end{array}$ \\
\hline
\end{tabular}

In the third stage, after screening the articles through the IC, the verification and removal of duplicate papers were carried out. Besides, a filtering procedure-by considering title, abstract, and keywords-was performed to exclude papers that did not present specific terms related to the theme of this review. Such a process was guided by the exclusion criteria (EC) (see Table 3) and was executed through the Rayyan web application [38].

Table 3 Exclusion criteria

\begin{tabular}{ll}
\hline EC & Description \\
\hline 01 & Duplicate articles \\
02 & $\begin{array}{l}\text { Studies not related to the processing of biomedical sig- } \\
\text { nals, ML, smart systems and data analysis of patients } \\
\text { with ALS }\end{array}$ \\
\hline
\end{tabular}


In the fourth stage, the total reading of the filtered articles was performed. Hence, it was executed the quality assessment (QA) protocol (see criteria in Table 4). In the QA procedure, each criterion was attributed points measuring the relevance of the article to the target subject of this research. The points were distributed in the form of weights $(w)$, considering suitable responses to the QA criteria, present in the primary studies, with 1.0 being the most relevant weight and 0 the lowest:

$$
w_{\mathrm{QA}}=\left\{\begin{array}{l}
1.0, \text { yes, fully describes, } \\
0.5, \text { yes, partially describes } \\
0, \text { does not describe }
\end{array}\right.
$$

A score, the arithmetic mean of the points of the QA criteria (Eq. 1), was generated for each article. In this case, all articles that obtained a score greater than or equal to 0.5 $(0.5 \leq$ score $\leq 1)$ were selected for this research and constitute the final set of articles.

$$
\text { score }=\frac{1}{\mathrm{QA}} \sum_{i=1}^{\mathrm{QA}} w_{\mathrm{QA}_{i}}
$$

Records relevant to each stage, as well as the data extracted from the articles, were properly gathered in spreadsheets and the Rayyan web application [38] for data extraction. Data, such as year of publication, authors, and possible responses to the RQ, were extracted from the set of articles of the fourth stage. They permitted the final analysis and fulfillment of the objectives of this systematic review.

\section{Results}

The results obtained from the searching and screening process of primary studies are synthesized in Fig. 2. In the first stage, 10128 candidate articles were identified after searching with the STRs. In the subsequent phase, three refining procedures based on IC (Table 2) were applied, and 9914 papers were discarded for not meeting the IC. At this point, 214 articles were considered appropriate for inclusion and analysis in the following stage. In stage three, the applied filters, based on the EC (Table 3), removed 186 articles amongst duplicates and those missing the target terms of the search. In this manner, 28 studies were selected for full-text reading and assessment through the QA criteria. After the QA procedure, the fourth and latter phase, 18 papers exceeded the pre-established minimum score, according to the result presented in the respective column in Table 5, and were included for analysis and definitive investigation in this review.

Table 4 Quality assessment

\begin{tabular}{ll}
\hline QA & Description \\
\hline 01 & Does the study clearly describe the types of biomedical signals? \\
02 & Does the study describe how signal processing is performed (algorithmic techniques, intelligent systems)? \\
03 & $\begin{array}{l}\text { Does the study describe the process of the proposed application for ALS patients (does it detail how it was } \\
\text { applied)? }\end{array}$ \\
04 & Does the study clearly describe its scientific contributions to the evolution of ALS-related research?
\end{tabular}




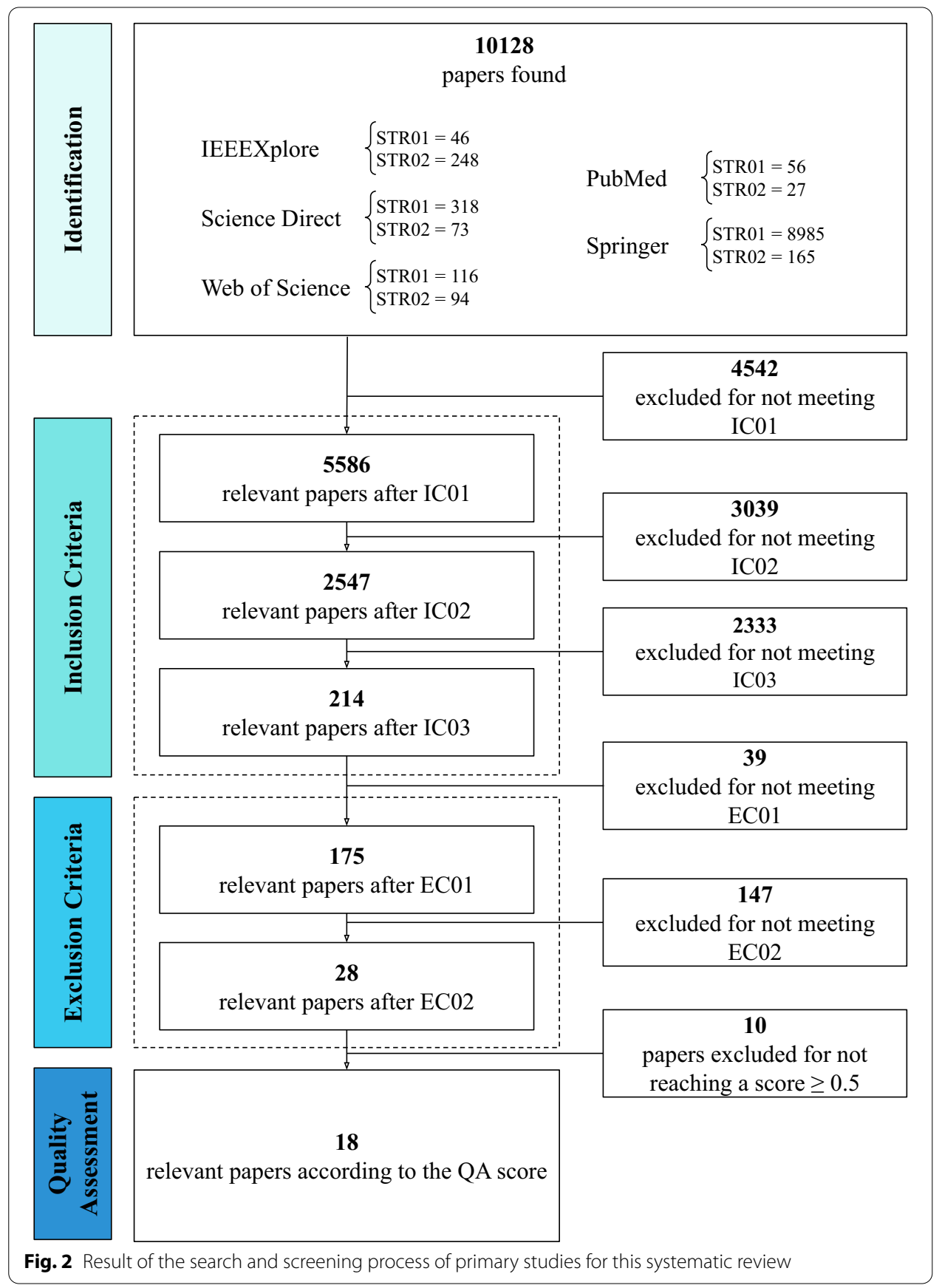

In sum, considering the 18 articles included in this research, the results presented in Fig. 3 evidence three major classes of probable practical applications of biomedical signals processing and machine learning within the context of the ALS disease: diagnosis (or classification), communication, and survival prediction. In addition to categorizing the purposes of such studies, Fig. 3 highlights the number of biomedical signals used and the respective classes that utilized them. Four distinct types of signals were identified: EMG, EEG, GR, and MRI. 


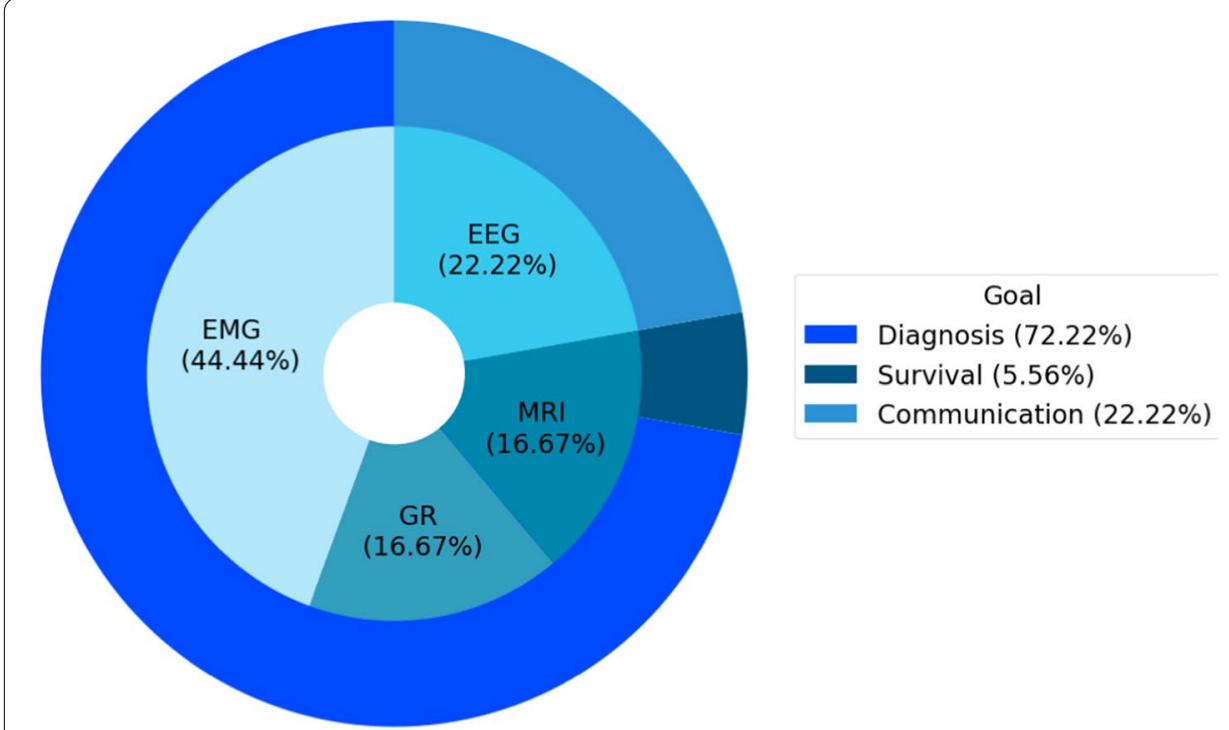

Fig. 3 Summary of the signals used and their objectives

Of the analyzed studies, $44.44 \%$ focus on the processing of the EMG signal, the most used biomedical signal (see Fig. 3), and specifically for classification. That is, for the diagnosis of individuals amongst healthy controls (HC), ALS patients, and, in some cases, other diseases (OD). With the same objective, especially for classification, $16.67 \%$ of the studies use GR and $11.11 \%$ MRI. The MRI signal was additionally used in a particular article for survival prediction of ALS-afflicted individuals, which represents $5.56 \%$. In the communication class, $22.22 \%$ of the studies focus exclusively on the approach through the processing of the EEG signal, being this the only one presented for that purpose. This first general analysis of the studies, identifying the purposes of the articles and the signals used, answers research questions RQ01 and RQ02.

Other significant and specific characteristics extracted from the 18 articles included in this study are summarized in Table 5, to support the analysis and answer research questions RQ03, RQ04, and RQ05. Regardless of the classes observed, diagnosis, communication, or survival prediction, all studies used ML algorithms. Alternative algorithmic models were employed and, according to the performance analysis of the algorithm concerning accuracy (Acc), specificity (Spe), or sensitivity (Sen) metrics of evaluation, the best or the only proposed model of each work is shown in Table 5, as well as their respective performances.

For testing, validating, and appraising the proposed approaches in the studies, the algorithmic techniques were applied to a set of data from individuals, distributed in different group combinations of HC, ALS, and/or myopathy, or other neurological diseases. The number of individuals and the type of participating groups in the experiments of each study is specified in Table 5 and summarized in Fig. 4. Moreover, Table 5 describes the source of the dataset and specifies whether they come from public or local repositories. 


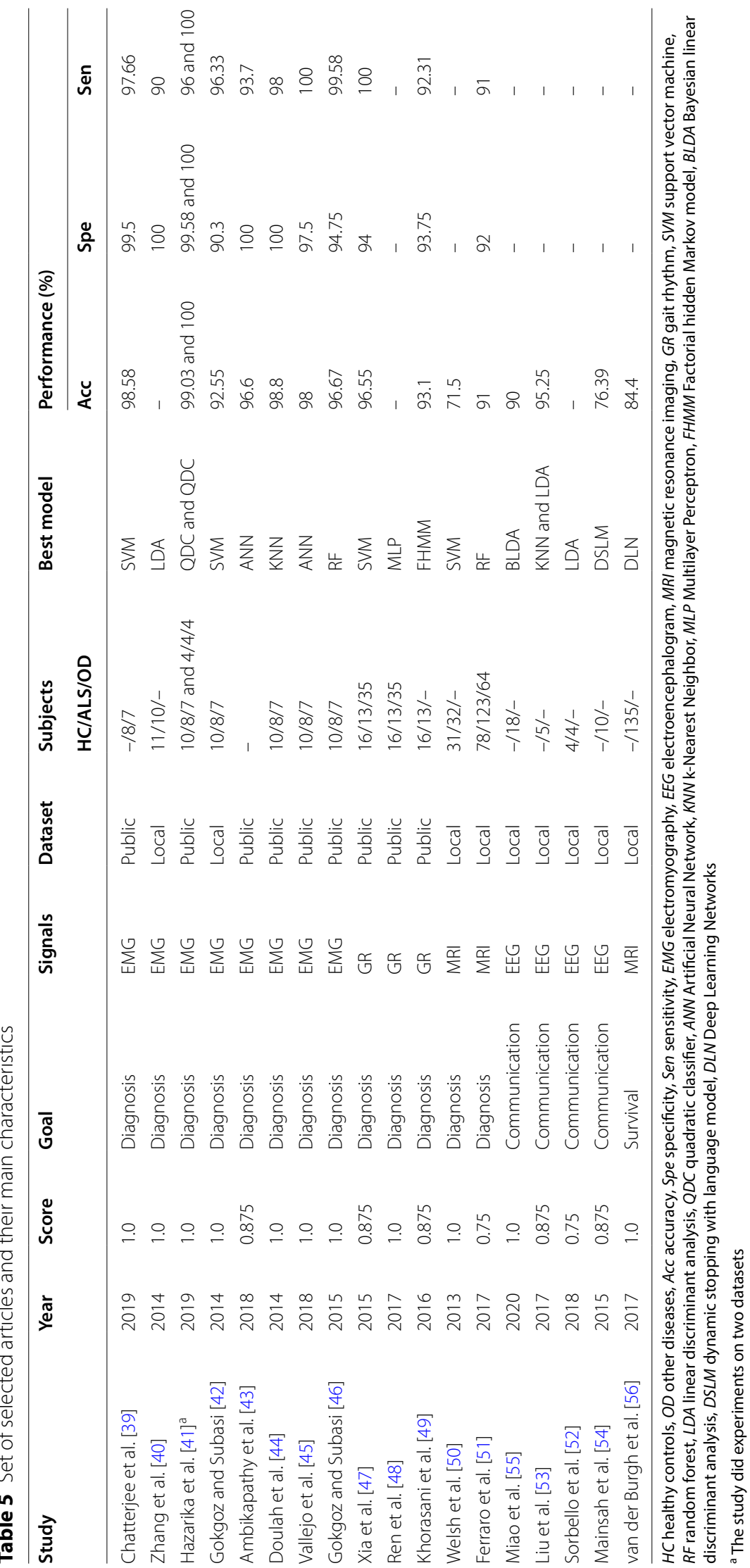




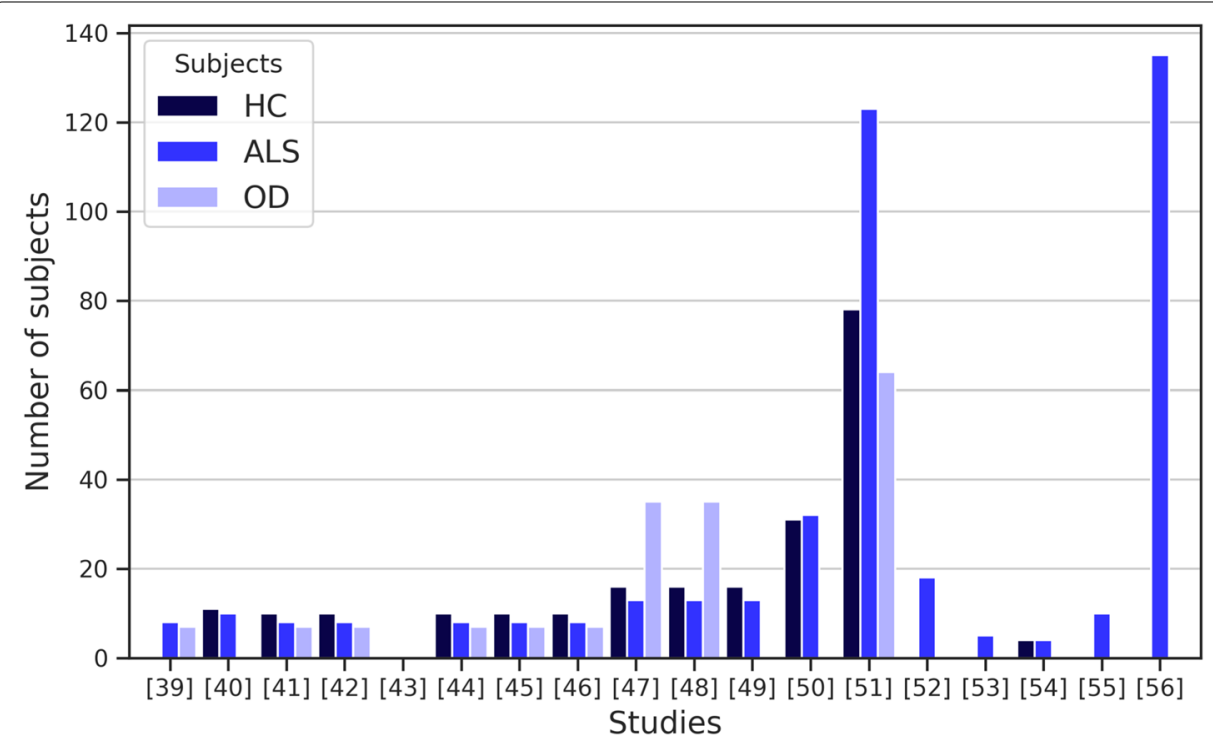

Fig. 4 Number of individuals used in the studies

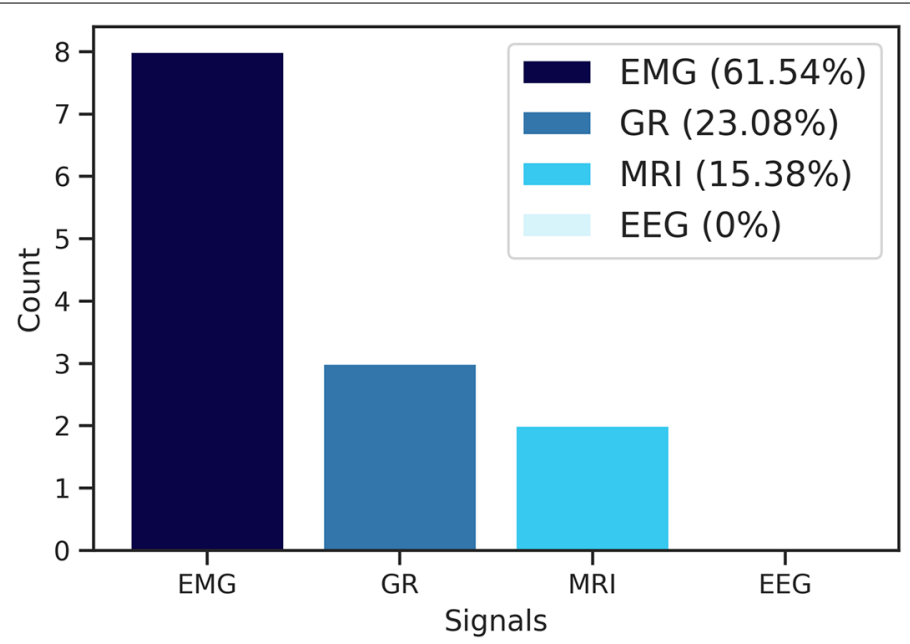

Fig. 5 Number of studies by type of biomedical signal defined in the diagnosis class

\section{Description of the diagnosis studies}

Diagnosis of ALS patients is the most numerous task described among the selected papers, accounting for $72.22 \%$ of the studies. Considering only this class, Fig. 5 presents an overview of the number of biomedical signals employed. The use of the EMG signal stands out, being addressed in $61.54 \%$ of the studies aimed at diagnosis [3946]. GR is applied in $23.08 \%$ of the studies [47-49] and MRI in $15.38 \%$ [50, 51]. The EEG signal is unused for this purpose.

In the studies, the focus on the development of strategies to reduce the noise of collected biomedical signals is evident, intending to discover the most significant features to enhance the performance of the algorithmic models, therefore reflecting on the accurate classification of individuals. In the case of the EMG signal, Gokgoz and 
Subasi [42] show the performance gain in the classification of HC, ALS, and OD with different ML algorithms, such as KNN, ANN, and SVM, after applying the multiscale principal component analysis (MSPCA) noise removal technique combined with the multiple signal classification (MUSIC) feature extraction technique.

In this study, the most satisfactory performance was of the SVM model, with $92.55 \%$ Acc. In the following year, also addressing the EMG signal, Gokgoz and Subasi [46] proposed another study focusing on noise reduction and selection of features. Hence, in this research, the authors developed a structure to eliminate noise, also employing the MSPCA technique, with a novel strategy based on Discrete Wavelet Transform (DWT) for feature extraction. The authors performed experimental tests, with and without the noise-removing structure and feature extraction, utilizing three different DT algorithmic models: CART, C4.5, and random forest (RF). Similarly to their previous study, Gokgoz and Subasi [46] achieved satisfactory results in their second work in the classification of HC, ALS, and OD, and demonstrated that the use of the MSPCA noise removal method in conjunction with feature extraction using DWT improved the performance of the RF algorithmic model, which obtained in the best case 96.67\% Acc using the EMG signal. In both studies, three distinct groups of subjects were considered: $\mathrm{HC}=10, \mathrm{ALS}=8$ and $\mathrm{OD}=7$.

Vallejo et al. [45] adopted the DWT approach to decompose EMG signals and generate the hyperspace of features that aims at selecting the most relevant features through the fuzzy entropy technique to feed an ML algorithm based on an ANN. To verify and validate the proposed method, the authors used a feedforward ANN with four layers of seven neurons each, except for the output layer, and the log-sigmoid activation function. The ANN, acting with the DWT and fuzzy entropy approaches, obtained 98\% Acc in the best result. Such a fact indicated that the proposed strategies for feature selection and extraction improved the classification process of individuals into three distinct groups, including HC, ALS, and/or myopathy. In the experiments, the authors included ten healthy subjects, eight with ALS and seven with OD.

In Doulah et al. [44], the DWT approach was applied to decompose, filter, and extract relevant features from preprocessed EMG signals. Preprocessing encompassed two methods proposed for the classification of subjects in HC and ALS, and/or myopathy. In the direct EMG method with DWT, features are extracted after a frame-by-frame sequential analysis. For the second approach, a set of features was selected through the procedure of dominant motor unit action potential (MUAP), and, based on the DWT decomposition, the key features for classification were extracted. Doulah et al. [44] verified both proposed methods in a KNN classifier. The authors evaluated the performance of the algorithm by operating distinct settings on three separate sets of data corresponding to $\mathrm{HC}$, ALS, and OD subjects (10, 8 and 7 subjects, respectively). Both processes presented significant results for the classification of two and three sets (HC, ALS, and OD). The Dominant-MUAP method may be highlighted for its consistent performance, reaching out $98.8 \%$ Acc using three sets.

Chatterjee et al. [39] presented a new generalization of the Stockwell transform (ST), called Modified Window ST (MWST), for preprocessing the EMG signals to generate a more representative feature matrix (or a time-frequency plane). MWST parameters $\alpha, \beta, \gamma$, and $\delta$ which affect the shape of the window or the energy concentration in the 
time-frequency plane, are defined in an optimized way through the Particle Swarm Optimization (PSO) metaheuristic algorithm. After applying the MWST technique to the EMG signals of eight individuals with ALS and seven with myopathy, four features are extracted from the matrix to serve as input to four ML models: SVM, KNN, Naïve Bayes, and DT. The approach proposed by Chatterjee et al. [39] presented significantly better results in the classification of individuals between ALS and myopathy when compared to the conventional ST method. The SVM model achieved the most satisfactory performance with $98.28 \%$ Acc.

Four distinct methods of feature extraction from EMG signals in the time-frequency domain have been addressed ST, Synchro-extracting Transform, Wigner Ville distribution, and Short-Time Fourier Transform. The four approaches applied to EMG signals generated images and, using the Gray Level Co-occurrence Matrix technique, 20 features were extracted with each method, and a set of 80 features was originated. To optimize the performance of the ML model proposed by the authors, an ANN with a hidden layer of 10 neurons and tan sigmoid activation function combined with a subset containing the most suitable combination of 15 features (from a finite set of possibilities) was defined through a genetic algorithm (GA), implemented with the KNN classifier to carry out fitness evaluation of the different combinations of the GA population. The strategy proposed by Ambikapathy et al. [43] to aid diagnosis using ANN obtained promising and statistically significant results in the process of classifying individuals between $\mathrm{HC}$ and ALS (86.6\% Acc, 86.6\% Spe, 86.6\% Sen), HC, ALS or myopathy (82.2\% Acc, 81.89\% Sen, $91.31 \%$ Spe), and ALS or myopathy (96.6\% Acc, $93.7 \%$ Sen, $100 \%$ Spe).

Zhang et al. [40] developed a method to characterize patterns in surface EMG signals based on three markers/features for supporting the diagnostic: clustering index, kurtosis of the EMG signal amplitude histogram, and kurtosis of EMG zero crossing-rate expansion. Furthermore, the linear discriminant analysis (LDA) ML algorithm was applied to the process of discriminating subjects as HC and ALS, obtaining as input data a feature vector originated from the concatenation of vectors of the mentioned features. The experiments carried out in this study relied on the analysis of data from 10 subjects with ALS and 11 from HC. Each of the three features displayed unique promising results. When used synergistically in an approach that combines them with the LDA algorithm, it rendered a more robust technique that presents even more significant outcomes, presenting a Spe of $100 \%$ and Sen of $90 \%$. Such an aspect indicates favorable perspectives to be used as a diagnosis support application.

Hazarika et al. [41] proposed a novel process assessment and inference system (PAIS) with a robust structure for preprocessing and extracting features from EMG signals. This structure is composed of procedures that initially evaluate the EMG signal features via an approach involving partitioning strategies of the input data (called multi-view direct) and decomposition by DWT, followed by the application of multidomain multiview discriminant correlation analysis ( $\mathrm{mmDCA}$ ). mmDCA analyzes the correlation of features, verifies redundancy, eliminates irrelevant features, and synchronizes them from partitions in a single vector. The mmDCA produced vector is incorporated into different ML models, such as KNN, and fed to a feedforward back-propagation neural network (FFBP-ANN) with two hidden layers of 8 neurons each, a conventional density-based linear classifier (LDC), a quadratic classifier (QDC) and SVM. 
Each algorithm performance was evaluated positively in the classification of subjects between HC, ALS, and myopathy classes in two real-time EMG signal datasets. In the first dataset, containing ten healthy subjects, eight with ALS and seven with OD, and in the second dataset, containing four healthy subjects, four with ALS and four with OD, the QDC model presented the most robust results: 99.03\% Acc and 100\% Acc, respectively.

The studies mentioned thus far show the potential and the importance of processing the EMG biomedical signal to provide consistent and elementary features or data to the learning of intelligent algorithms and, consequently, to help with the diagnosis of ALS. However, as an alternative to EMG signals for diagnosis, the studies [47-49] investigated the performance of ML models with strategies that process data from GR biomedical signals.

A study by Xia et al. [47] carried out feature extraction from GR signals in five time series records, based on statistical analysis. Features such as mean value, standard deviation, maximum and minimum value, skewness, and kurtosis were computed and defined. The authors also proposed the extraction of three more features using the Lempel-Ziv complexity, fuzzy entropy, and Teager-Kaiser energy operator statistical methods. Furthermore, Xia et al. [47] executed an approach of selecting a subset of features based on three procedures, with the first being a statistical analysis of the features, followed by a performance evaluation of classification algorithms and ultimately the application of the hill-climbing optimization algorithm to find out and define the optimal subset of features.

After selection, a series of experiments with ML algorithms was performed considering the optimal subset as the input data for four classifiers: SVM, RF, a feedforward ANN with sigmoid activation function, and KNN. The dataset used for the experiments included data from 16 healthy subjects, 13 with ALS and 35 with OD. All classifiers showed good performance in the binary classification between HC and ALS, and HC and OD (neurodegenerative) in addition to ALS. The most satisfactory performance was obtained by the SVM technique, with $96.55 \%$.

Ren et al. [48] performed a strategy to extract and select features from five time series of GR signals. For this, the researchers proposed an approach that utilizes the empirical mode decomposition (EMD) method to extract features from the partitioning of the GR signal time series, therefore producing six components-five of which are used and one discarded-that were promptly submitted to statistical analysis using Kendall's Coefficient of Concordance method. With this, the purpose is to measure the significance and relationship of the features. Next, a calculation that employed the amplitudes of the components was made through the procedure of Ratio for Energy Change. Such a process comprehends to a dimensionality reduction technique based on principal component analysis (PCA) is applied to define the final set of features.

The strategy proposed by the authors was evaluated in five classifiers: Naïve Bayes, SVM, RF, Multilayer Perceptron (MLP) and Simple Logistic Regression. Unlike the performance evaluation of the ML models considered in Table 5 , in this study the area under the ROC curve (AUC) is used for performance assessment. The most significant results for classifying subjects between HC and ALS (16 and 13 subjects, respectively) were presented by the feedforward MLP model, with an AUC value of 0.934. The average 
value of the AUC considering the five classifiers was 0.898 and, in general, the approach indicates promising results.

Differently from other researches developed for diagnostic, in the study of Khorasani et al. [49], a generalization of the Hidden Markov Model (HMM) and the classification model named the factorial hidden Markov model (FHMM), the recognition of patterns and the classification of subjects in HC or ALS from GR time series is proposed. After preprocessing the signal to remove outliers, the GR signal is segmented into fifty time series. And some features, such as mean or variance, are extracted to feed the FHMM. The method was assessed with data from 16 subjects with HC and 13 with ALS. Further, its performance was compared to the traditional HMM model and the Least-Squares SVM (LS-SVM) algorithm with Gaussian kernels. With an Acc value of 93.1\%, the FHMM classification model displayed superior performance in HC or ALS recognition.

Scarcely explored in the context of diagnosing patients with ALS, strategies based on neuroimaging and ML are still challenging. In 2013, Welsh et al. [50] proposed an approach when implementing the SVM algorithm to classify subjects in HC or ALS through the analysis of data from functional magnetic resonance imaging (fMRI). The fMRI time series data were preprocessed and underwent a set of robust procedures for the extraction and selection of features, including the consecutive execution of strategies, such as PCA and Independent Components Analysis (ICA), as well as the creation of maps (or vectors) of correlation coefficients from different brain regions. After that, the data were provided to the linear kernel SVM algorithm, and its performance was evaluated. For the experiments, data from 32 patients diagnosed with ALS and 31 people from $\mathrm{HC}$ were provided. Welsh et al. [50] indicated the values were modest (71.5\% Acc) in the classification of diseases as ALS using fMRI at rest.

A few years after the fMRI study by Welsh et al. [50], Ferraro et al. [51] developed a method for classifying individuals with motor neuron diseases, including ALS, based on the multimodal structural MRI with an ML algorithm. The MRI data were divided into distinct regions of interest and underwent analysis using literature and statistics software. The evaluation of the proposed diagnosis approach was performed in an RF model. The performance of the algorithm in classifying individuals in HC and ALS, specifically with the combined MRI model, was expressive. For the experiments, data from 78 individuals from HC, 123 subjects with ALS, and 64 subjects with OD were used. The model developed showed an Acc of 91\% concerning the classification of subjects with ALS and subjects from HC. The results of both studies [50,51] typically indicate that only data from MRI-based strategies are insufficient to obtain good performance in the classification. Studies with neuroimaging and ML for ALS are yet restricted. Nevertheless, the results are promising for the development of a system capable of assisting in ALS diagnosing.

\section{Communication improving studies}

As a result of the progressive degeneration of the upper and/or lower motor neurons in the brainstem region, people diagnosed with ALS lose the ability to speak and interact with the environment. Technological resources developed for communication are crucial to ensure the well-being of those patients. About $22 \%$ of the studies included in this 
review [52-55], have developed artifacts that promote the communication improvement class of this SLR, exclusively through the EEG biosignal.

In a study conducted by Sorbello et al. [52], a framework was proposed through the operation of a brain-computer interface (BCI) system to control a humanoid robot and promote minimal autonomy to patients with ALS. Generally, the system structure, called brain-computer robotic interface (BCRI), is composed of a BCI system, EEG and eyetracking devices, and a network system to connect the $\mathrm{BCI}$ system to the robotic system. The ML LDA algorithm is used after preprocessing and EEG feature extraction to correctly classify and translate the user action into control commands for the humanoid robot. The authors evaluated the proposal by conducting experiments on four subjects at the $\mathrm{HC}$ and four subjects with ALS. The results were satisfactory, and the proposed framework for enabling communication for patients with ALS was validated after all participants were able to control the humanoid robot.

Liu et al. [53] developed an approach by applying the concepts of fractal dimension (FD) and Fisher's criterion to optimize the selection of EEG channels and the characterization of the data obtained from the signal. In this manner, the authors aimed at improving the classification capacity of an ML algorithm in a BCI system for patients with ALS. Two methods for estimating FD, Grassberger-Procaccia (GPFD) and Higuchi (HFD), were implemented. The key features of 30 EEG channels were extracted and concatenated into a single vector to serve two algorithmic models: KNN and LDA. After tests performed on five subjects with ALS, the results were satisfactory and the GPFD method surpassed the HFD. The performances of the two algorithms, KNN and LDA, were significant and similar, with $95.25 \%$ Acc, when compared with the input data containing the 30 EEG channels.

The existence of a simple interface with an accurate and fast information transfer rate is essential to maintain communication efficiency in a BCI system based on EEG signals for people with ALS. For addressing such matter, Mainsah et al. [54] developed a data-driven Bayesian early stopping algorithm, called DS, to optimize the feature selection process of an ERP-based P300 BCI speller, in which ERP stands for event-related potentials. Besides, a variation of the DS is proposed with the application of statistical modeling through Bayesian inference for language predictability, called DSLM. Features correlated with the user's interest were extracted from the EEG signal to train the stepwise LDA classifier. In the research, the designated online tests were performed with 10 subjects with ALS. Both DS and DSLM algorithms proved to be efficient in minimizing the character selection time and with an average accuracy of $75.40 \%$ and $76.39 \%$, respectively. There was no statistical difference between the algorithms.

In the same context of Mainsah et al. [54] and Miao et al. [55] proposed an ERP-based $\mathrm{BCI}$ display approach using as strategy a new speller paradigm with peripherally distributed stimuli with the possibility of feedback in the center of the display. The EEG signals were recorded and analyzed using preexisting software from the BCI platform. The features were extracted from data acquired offline from 16 electrodes to train the Bayesian LDA (BLDA) classifier and subsequently utilization of the trained model in an online system test. The proposed method was evaluated concerning the conventional matrix speller paradigm. The experiments were carried out on 18 subjects with ALS. Even obtaining an Acc of $90 \%$ in its most efficient performance, the results presented by the 
BLDA algorithm do not reveal any significant difference between the proposed approach and the conventional approach. However, patients with ALS were able to operate the system effectively.

\section{Survival prediction studies}

The survival prediction of patients with ALS is empirically defined based on, generally, the analysis of clinical data. Only one of the studies included in this review is dedicated to such a prediction. In the study developed by van der Burgh et al. [56], a model for predicting survival (short, medium, or long) of patients with ALS is proposed by combining clinical data, neuroimaging, and a robust ANN-based ML technique named Deep Learning Networks (DLN). Four scenarios were defined for the application of the DLN algorithm. Finally, the first situation was based only on clinical data. The second and third scenarios utilized MRI images and included structural connectivity and brain morphology data. The latter situation included a combination of the previous three. To each of those, a model was implemented. Furthermore, the performance of algorithms was evaluated through a database that contained data of 135 subjects with ALS. The model that combined clinical and MRI data revealed superior performance $(84.4 \%$ of Acc value) and was presented as a viable strategy for predicting the survival of patients with ALS. The remaining models displayed intermediate results, although they indicated promising approaches.

\section{Discussions}

This systematic literature review explored approaches based on computational intelligence. Besides, to process biomedical signals considering the scope of ALS, it functioned in a synergistic and complementary manner. A set of 18 articles was included and reviewed, and three major classes of applications were found: aid to diagnostic, communication enabling, and survival prediction. The most adequate algorithmic models and the respective biomedical signals responsible for providing data were identified and quantified (see Fig. 6).

Based on the analysis of the 13 articles that addressed the support to the diagnosis of patients with ALS, regardless of the biomedical signal or ML algorithm used, it is possible to define a standard methodological scheme (a pipeline) general to all studies, which is broadly depicted in Fig. 7. Except for Khorasani et al. [49], who investigated a new classification algorithm, the studies suggest approaches or methods for the data treatment process that may enhance the training stage and, consequently, the classification stage. This data treatment process, which includes the feature extraction and selection phases, for instance, is important to eliminate noise, redundancy, and reduce the data dimensionality, in addition to maximizing the performances of the algorithms through the provision of refined and consistent data [57]. The various ML models implemented were presented as techniques for evaluating and validating the proposals of the studies. However, they were elementary techniques in the diagnosis process that are present in all articles. 


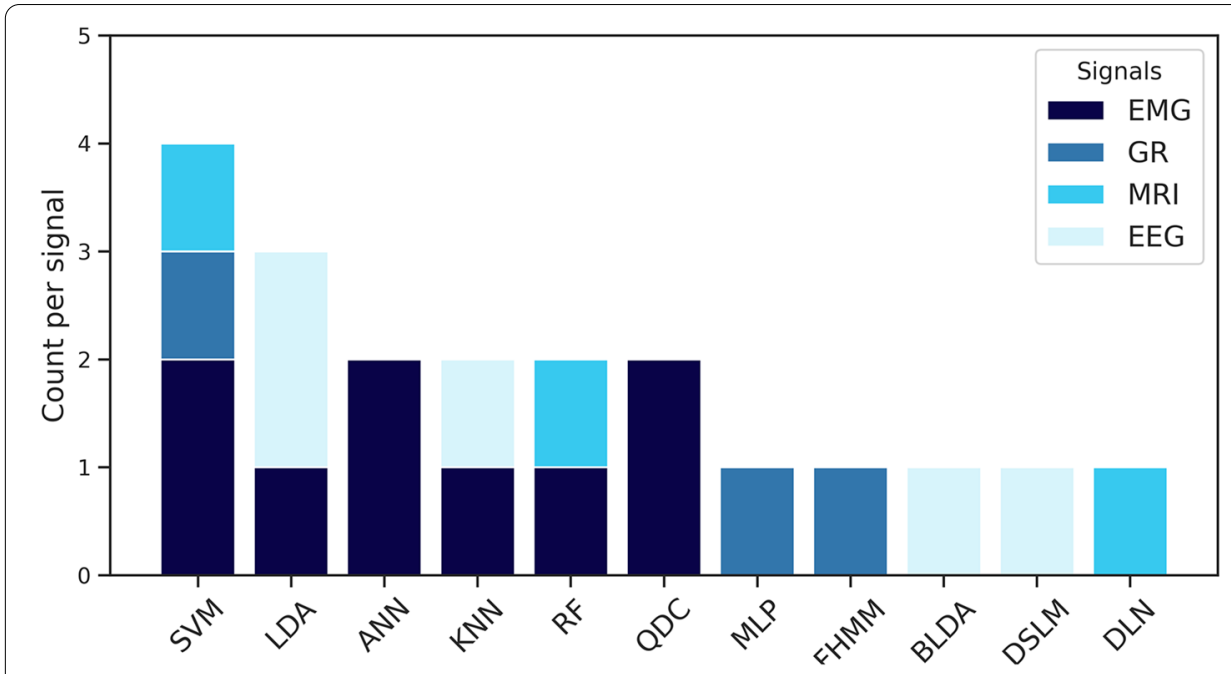

Fig. 6 Quantitative of the best algorithmic models and the respective biomedical signals

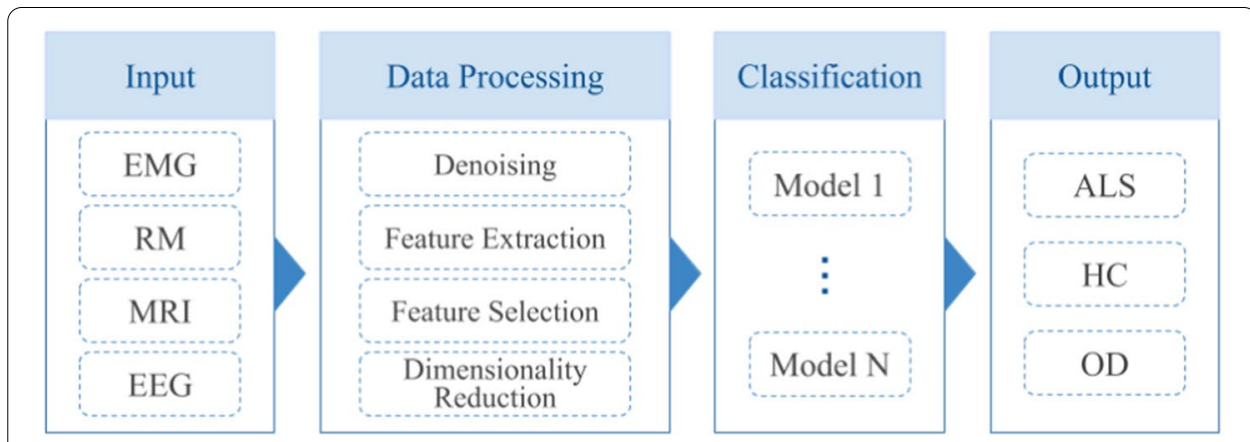

Fig. 7 Generic pipeline: generalized scheme for solving classification problems

The studies [39, 41-48, 51] about diagnosis support, in addition to ALS, also tested approaches for binary or multi-label classification considering other neurological conditions, such as myopathy, Parkinson's disease, Huntington's disease, predominantly upper motor neuron disease, and ALS-mimic disorders.

Regarding the four articles belonging to the communication class, two distinct categories can be observed in the analyzed studies. The first one is that of the study conducted by Sorbello et al. [52], which aims at complementing and adapting a BCI system with a humanoid robot to provide not only communication but also a minimum of autonomy. In the second identified category, the studies [53-55] suggest alternative approaches that include ML to optimize character selection time in a BCI system. These approaches range from the optimization of EEG electrodes to intelligent customization of the interface. The importance of BCI systems in promoting communication is evident. These systems are widely utilized in research to establish a communication pathway between the human brain and external devices, recognizing voluntary changes in the brain activity of their users [58-63].

Despite the research focused on the development of BCI systems, there are limitations regarding their home use. One of the primary reasons why $\mathrm{BCI}$ have not been 
introduced into the domestic environment is the character selection time. Specifically, the time still is considered slow and inaccurate when it comes to approaches that do not use brain signals, and also the need for electrodes connected to the head of the patient $[47,54,64]$. Other approaches to human-computer interaction systems that do not necessarily involve brain signals by EEG can be seen in Pinheiro et al. [65], Hori et al. [66], Fathi et al. [67], Harezlak et al. [68], Villanueva et al. [69], Królak e Strumiłło [24], Zhao et al. [70], Liu et al. [71] and Aharonson et al. [22].

The only survival prediction study with ALS patients analyzes how challenging it is to develop systems for such a purpose. The study [56] indicates that MRI and the DLN technique are promising for survival prediction and suggests a more significant exploration of the field of neuroimaging. Also, the research reveals the importance and benefits of patients' clinical data in the process of predicting survival at the three levels of ALS. This observation, in combination with the analysis made thus far, reveals both the absence and the possibility of using clinical data for diagnosis. Correlated with the survival aspect, recent studies indicate it is possible to apply ML approaches with digital biomarkers using the speech signal to monitor the progression of ALS [72], including applications for automatic classification of the ALS Functional Rating Scale (ALSFRS) [73, 74].

Regarding ML algorithms, it is observed that they are specifically supervised in all studies. The type of biomedical signal varies only in the diagnosis studies, with EMG being the most used signal, followed by GR and MRI. The EEG signal is applied solely for communication enabling applications. The MRI-based biomedical signal is used both in diagnosis and survival prediction applications. Schuster et al. [75] affirm that MRI-based biomarkers are currently seldom used for aiding the identification of ALS. This observation is complemented by the results presented in this SLR, which also reports the limited number of neuroimaging-based studies aimed at diagnosis support applications and survival prediction of the ALS disease, despite the potential mentioned by van der Burgh et al. [56]. In addition to these biomedical signs mentioned so far, studies show the feasibility of using the speech biosignal for the early diagnosis of ALS, as indicated by Wang et al. [76], Suhas et al. [77], An et al. [78], Vieira et al. [79], and Wisler et al. [80], and tracking changes in individuals with bulbar ALS [81].

The 18 studies carried out experimental tests with datasets of healthy subjects and subjects with ALS or other neurological diseases. $50 \%$ of the studies used local or proprietary datasets. The other $50 \%$ of the investigations collected data from public online repositories. In some cases, like those for diagnosis and communication, except in the study carried out by Ferraro et al. [51], the limitation in the number of patients with ALS is evident (see Fig. 4). These results suggest that it is still challenging to develop and validate a robust study with a more considerable number of subjects with ALS or in an outpatient setting.

\section{Conclusion}

This article introduces an SLR protocol to investigate relevant studies from the last ten years (2009-2019) that address ML techniques and biomedical signal processing. It may contribute to the advancement of research within the context of ALS. Based on 18 primary studies, the results exhibit strategies to minimize problems and/or promote means 
for diagnosis support, communication, and survival prediction. Considering the analyzed studies, $88.89 \%$ of those report the importance of treating biomedical signals for providing robust and consistent data for ML algorithmic models.

Furthermore, it can be observed that there is a predominance in the type of biomedical signals used by studies in the categories of communication and prediction of survival, being exclusively and respectively the EEG signals and MRI images. For the diagnosis class, in particular, three types of raw data are reported, namely EMG (61.54\%), GR (23.08\%), and MRI (15.38\%). Regarding ML algorithmic models and analyzing the most satisfactory performances, SVM is the most used, followed by LDA and ANN techniques. Even though the 18 articles selected use ML, except for one study that proposed a new algorithm. In general, limited to the objectives of this SLR, the literature suggests and dedicates itself to the treatment of biomedical signals.

The studies are promising, but there are, nonetheless, significant aspects to be explored. When it comes to the diagnosis, the studies may be applied in outpatient clinics for practical assistance, in cases that have yet been unconfirmed of ALS, or even so in the early stages of the disease. Moreover, the use of big data approaches with patient's clinical data might contribute to the conclusive results and remains open for investigations. That includes the field of survival prediction. Concerning the approaches for communication improvement, there are unanswered questions about the use of $\mathrm{BCI}$ in the domestic environment, considering its aspects, costs, as well as efficient interfaces that prevent fatigue, discomfort, and optimization of the electrodes for EEG signals acquisition.

\begin{abstract}
Abbreviations
Acc:: Accuracy; ALS:: Amyotrophic lateral sclerosis; ALSFRS: ALS Functional Rating Scale; ANN:: Artificial Neural Network; AUC:: Receiver operating characteristic curves; $\mathrm{BCl}:$ : Brain computer interface; BCRl:: Brain computer robotic interface; BLDA:: Bayesian linear discriminant analysis; DLN:: Deep Learning Network; DS:: Dynamic stopping; DSLM:: Dynamic stopping with language model; DT:: Decision tree; DWT:: Discrete Wavelet Transform; EC:: Exclusion criteria; ECG:: Electrocardiogram; EEG:: Electroencephalogram; EMD:: Empirical mode decomposition; EMG:: Electromyography; EOG:: Electrooculogram; FD:: Fractal dimension; FHMM:: Factorial hidden Markov model; fMRI:: Functional magnetic resonance imaging; GA:: Genetic algorithm; GPFD:: Grassberger-Procaccia fractal dimension; GR:: Gait rhythm; HC:: Healthy controls; HFD:: Higuchi fractal dimension; HMM:: Hidden Markov model; IC:: Inclusion criteria; ICA:: Independent components analysis; KNN:: K-Nearest Neighbor; LDA:: Linear discriminant analysis; LDC:: Density-based linear classifier; LS-SVM:: Leastsquares support vector machine; ML:: Machine learning: MLP:: Multilayer perceptron; mmDCA:: Multidomain multiview discriminant correlation analysis; MRI:: Magnetic resonance imaging; MSPCA:: Multiscale principal component analysis; MUAP:: Motor unit action potential; MUSIC:: Multiple signal classification; MwST:: Modified window Stockwell transform; OD:: Other diseases; PAIS:: Process assessment and inference system; PCA:: Principal component analysis; PSO:: Particle Swarm Optimization; QA:: Quality assessment; QDC:: Quadratic classifier; RF:: Random forest; RQ:: Research questions; Sen:: Sensitivity; SLR:: Systematic literature review; Spe:: Specificity; ST:: Stockwell transform; STR:: Search strings; SVM: Support vector machine and $(w)$ : weights,
\end{abstract}

\title{
Acknowledgements
}

We kindly thank the Laboratory of Technological Innovation in Health (LAIS) of the Federal University of Rio Grande do Norte (UFRN) and Ministry of Health Brazil for supporting the research.

\section{Authors' contributions}

$\mathrm{FF}, \mathrm{B}$ and $\mathrm{DB}$ : collection, organizing and review of the literature. FF and IB: preparing and edit the manuscript. FF, IB, DB, $\mathrm{RV}, \mathrm{CT}, J \mathrm{H}, \mathrm{PG}$ and $\mathrm{MDJ}$ : manuscript review and modification. All authors read and approved the final manuscript.

Funding

Ministry of Health Brazil.

Data availability

Not applicable. 


\section{Declarations}

Ethics approval and consent to participate

Not applicable.

\section{Consent for publication}

Not applicable.

\section{Competing interests}

The authors declare that they have no competing interests.

\section{Author details}

${ }^{1}$ Laboratory of Technological Innovation in Health (LAIS), Federal University of Rio Grande do Norte (UFRN), Natal, RN, Brazil. ${ }^{2}$ Department of Informatics Engineering, Univ Coimbra, CISUC-Center for Informatics and Systems of the University of Coimbra, Coimbra, Portugal.

Received: 3 December 2020 Accepted: 9 June 2021

Published online: 15 June 2021

\section{References}

1. Saadeh W, Altaf MAB, Butt SA. A wearable neuro-degenerative diseases detection system based on gait dynamics. In: 2017 IFIP/IEEE international conference on very large scale integration (VLSI-SoC). 2017. p. 1-6. https:// doi.org/10.1109/VLSI-SOC.2017.8203488.

2. Hardiman O, Al-Chalabi A, Chio A, Corr EM, Logroscino G, Robberecht W, Shaw PJ, Simmons Z, van den Berg LH. Amyotrophic lateral sclerosis. Nat Rev Dis Prim. 2017;3(1):17071. https://doi.org/10.1038/nrdp.2017.71.

3. van Es MA, Hardiman O, Chio A, Al-Chalabi A, Pasterkamp RJ, Veldink JH, van den Berg LH. Amyotrophic lateral sclerosis. Lancet. 2017;390(10107):2084-98. https://doi.org/10.1016/S0140-6736(17)31287-4.

4. Chiò A, Pagani M, Agosta F, Calvo A, Cistaro A, Filippi M. Neuroimaging in amyotrophic lateral sclerosis: insights into structural and functional changes. Lancet Neurol. 2014;13(12):1228-40. https://doi.org/10.1016/S1474-4422(14) 70167-X.

5. Lima SR, Gomes KB. Esclerose lateral amiotrófica e o tratamento com células-tronco. Rev Bras Clin Med. 2010;8(6):531-7.

6. Kiernan MC, Vucic S, Cheah BC, Turner MR, Eisen A, Hardiman O, Burrell JR, Zoing MC. Amyotrophic lateral sclerosis. Lancet. 2011;377(9769):942-55. https://doi.org/10.1016/S0140-6736(10)61156-7.

7. Turner MR, Hardiman O, Benatar M, Brooks BR, Chio A, de Carvalho M, Ince PG, Lin C, Miller RG, Mitsumoto H, Nicholson G, Ravits J, Shaw PJ, Swash M, Talbot K, Traynor BJ, Van den Berg LH, Veldink JH, Vucic S, Kiernan MC. Controversies and priorities in amyotrophic lateral sclerosis. Lancet Neurol. 2013;12(3):310-22. https://doi.org/10.1016/S14744422(13)70036-X.

8. Andersen PM, Abrahams S, Borasio GD, de Carvalho M, Chio A, Van Damme P, Hardiman O, Kollewe K, Morrison KE, Petri S, Pradat P-F, Silani V, Tomik B, Wasner M, Weber M, The EFNS Task Force on Diagnosis and Management of Amyotrophic Lateral Sclerosis. EFNS guidelines on the clinical management of amyotrophic lateral sclerosis (MALS)—revised report of an EFNS task force. Eur J Neurol. 2012;19(3):360-75. https://doi.org/10.1111/j.1468-1331. 2011.03501.x

9. Brown RH, Al-Chalabi A. Amyotrophic lateral sclerosis. N Engl J Med. 2017;377(2):162-72. https://doi.org/10.1056/ NEJMra1603471.

10. Scarafino A, D’Errico E, Introna A, Fraddosio A, Distaso E, Tempesta I, Morea A, Mastronardi A, Leante R, Ruggieri M, Mastrapasqua M, Simone IL. Diagnostic and prognostic power of CSF Tau in amyotrophic lateral sclerosis. J Neurol. 2018;265(10):2353-62. https://doi.org/10.1007/s00415-018-9008-3.

11. Jeon G, Ahmad A, Cuomo S, Wu W. Special issue on bio-medical signal processing for smarter mobile healthcare using big data analytics. New York: Springer; 2019. https://doi.org/10.1007/s12652-019-01425-9.

12. Horton DK, Mehta P, Antao VC. Quantifying a nonnotifiable disease in the united states: the national amyotrophic lateral sclerosis registry model. JAMA. 2014;312(11):1097-8.

13. Goetz CG. Amyotrophic lateral sclerosis: early contributions of Jean-Martin Charcot. Muscle Nerve. 2000;23(3):336-43.

14. Arthur KC, Calvo A, Price TR, Geiger JT, Chio A, Traynor BJ. Projected increase in amyotrophic lateral sclerosis from 2015 to 2040. Nat Commun. 2016;7(1):1-6

15. Rosa Silva JP, Santiago Júnior JB, dos Santos EL, de Carvalho FO, de FrançCosta IMP, de Mendonça DMF. Quality of life and functional independence in amyotrophic lateral sclerosis: a systematic review. Neurosci Biobehav Rev. 2020;111:1-11. https://doi.org/10.1016/j.neubiorev.2019.12.032.

16. Bustamante P, Grandez K, Solas G, Arrizabalaga S. A low-cost platform for testing activities in Parkinson and ALS patients. In: The 12th IEEE international conference on e-Health networking, applications and services. 2010. p. 302-7. https://doi.org/10.1109/HEALTH.2010.5556550.

17. Bjornevik K, Zhang Z, O'Reilly ÉJ, Berry JD, Clish CB, Deik A, Jeanfavre S, Kato I, Kelly RS, Kolonel LN, Liang L, Marchand LL, McCullough ML, Paganoni S, Pierce KA, Schwarzschild MA, Shadyab AH, Wactawski-Wende J, Wang DD, Wang Y, Manson JE, Ascherio A. Prediagnostic plasma metabolomics and the risk of amyotrophic lateral sclerosis. Neurology. 2019;92(18):2089-100. https://doi.org/10.1212/WNL.0000000000007401.

18. Foerster BR, Dwamena BA, Petrou M, Carlos RC, Callaghan BC, Churchill CL, Mohamed MA, Bartels C, Benatar M, Bonzano L, Ciccarelli O, Cosottini M, Ellis CM, Ehrenreich H, Filippini N, Ito M, Kalra S, Melhem ER, Pyra T, Roccatagliata L, Senda J, Sobue G, Turner MR, Feldman EL, Pomper MG. Diagnostic accuracy of diffusion tensor imaging 
in amyotrophic lateral sclerosis: a systematic review and individual patient data meta-analysis. Acad Radiol. 2013;20(9):1099-106. https://doi.org/10.1016/j.acra.2013.03.017.

19. Al-Chalabi A, Hardiman O, Kiernan MC, Chiò A, Rix-Brooks B, van den Berg LH. Amyotrophic lateral sclerosis: moving towards a new classification system. Lancet Neurol. 2016;15(11):1182-94. https://doi.org/10.1016/S1474-4422(16) 30199-5.

20. Fraschini M, Lai M, Demuru M, Puligheddu M, Floris G, Borghero G, Marrosu F. Functional brain connectivity analysis in amyotrophic lateral sclerosis: an EEG source-space study. Biomed Phys Eng Express. 2018;4(3):037004. https://doi. org/10.1088/2057-1976/aa9c64.

21. Barbalho IMP, Silva PdA, Fernandes FRdS, Neto FMM, Leite CRM. An investigation on the use of ontologies for pattern classification —study applied to the monitoring of food intake. In: Proceedings of the Euro American conference on telematics and information systems. EATIS '18. New York: Association for Computing Machinery; 2018. https://doi.org/10.1145/3293614.3293627.

22. Aharonson V, Coopoo VY, Govender KL, Postema M. Automatic pupil detection and gaze estimation using the vestibulo-ocular reflex in a low-cost eye-tracking setup. SAIEE Afr Res J. 2020;111(3):120-4.

23. Lingegowda DR, Amrutesh K, Ramanujam S. Electrooculography based assistive technology for ALS patients. In: 2017 IEEE international conference on consumer electronics-Asia (ICCE-Asia). 2017. p. 36-40 . https://doi.org/10. 1109/ICCE-ASIA.2017.8307837.

24. Królak A, Strumiłło P. Eye-blink detection system for human-computer interaction. Univ Access Inf Soc. 2012;11(4):409-19. https://doi.org/10.1007/s10209-011-0256-6.

25. Höhne J, Holz E, Staiger-Sälzer P, Müller K-R, Kübler A, Tangermann M. Motor imagery for severely motor-impaired patients: evidence for brain-computer interfacing as superior control solution. PLoS ONE. 2014;9(8):104854.

26. Eid MA, Giakoumidis N, El Saddik A. A novel eye-gaze-controlled wheelchair system for navigating unknown environments: case study with a person with ALS. IEEE Access. 2016;4:558-73.

27. Schwalbe N, Wahl B. Artificial intelligence and the future of global health. Lancet. 2020;395(10236):1579-86. https:// doi.org/10.1016/S0140-6736(20)30226-9.

28. Subasi A. Classification of EMG signals using PSO optimized SVM for diagnosis of neuromuscular disorders. Comput Biol Med. 2013;43(5):576-86. https://doi.org/10.1016/j.compbiomed.2013.01.020.

29. Noorbakhsh-Sabet $N$, Zand R, Zhang $Y$, Abedi V. Artificial intelligence transforms the future of health care. Am J Med. 2019;132(7):795-801. https://doi.org/10.1016/j.amjmed.2019.01.017.

30. Grollemund V, Pradat PF, Querin G, Delbot F, Le Chat G, Pradat-Peyre JF, Bede P. Machine learning in amyotrophic lateral sclerosis: achievements, pitfalls, and future directions. Front Neurosci. 2019;13:135. https://doi.org/10.3389/ fnins.2019.00135.

31. AlHinai N. Chapter-introduction to biomedical signal processing and artificial intelligence. In: Zgallai W, editor. Biomedical signal processing and artificial intelligence in healthcare. Developments in Biomedical Engineering and Bioelectronics. Amsrerdam: Academic Press; 2020. p. 1-28. https://doi.org/10.1016/B978-0-12-818946-7.00001-9.

32. Cohen A. Chapter 1-biomedical signals: origin and dynamic characteristics; frequency-domain analysis. In: Bronzino JD, editor. Medical devices and systems. The Biomedical Engineering HandbookThe Biomedical Engineering HandbookThe Biomedical Engineering Handbook. Boca Raton: CRC Press; 2006. p. 1-22.

33. Alim OA, Moselhy M, Mroueh F. EMG signal processing and diagnostic of muscle diseases. In: 2012 2nd international conference on advances in computational tools for engineering applications (ACTEA). 2012. p. 1-6.

34. Luna P. Controlling machines with just the power of thought. Lancet Neurol. 2011;10(9):780-1. https://doi.org/10. 1016/S1474-4422(11)70180-6.

35. Chen S, Lach J, Lo B, Yang G. Toward pervasive gait analysis with wearable sensors: a systematic review. IEEE J Biomed Health Inform. 2016;20(6):1521-37. https://doi.org/10.1109/JBHI.2016.2608720.

36. Menke RA, Agosta F, Grosskreutz J, Filippi M, Turner MR. Neuroimaging endpoints in amyotrophic lateral sclerosis. Neurotherapeutics. 2017:14(1):11-23.

37. Kitchenham B. Procedures for performing systematic reviews. Technical report, Keele University, Department of Computer Science, Software Engineering Group and Empirical Software Engineering National ICT Australia Ltd., Keele, Staffs, ST5 5BG, UK; 2004.

38. Ouzzani M, Hammady H, Fedorowicz Z, Elmagarmid A. Rayyan — a web and mobile app for systematic reviews. Syst Rev. 2016;5(1):210. https://doi.org/10.1186/s13643-016-0384-4.

39. Chatterjee S, Samanta K, Choudhury NR, Bose R. Detection of myopathy and ALS electromyograms employing modified window Stockwell transform. IEEE Sens Lett. 2019;3(7):1-4. https://doi.org/10.1109/LSENS.2019.2921072

40. Zhang X, Barkhaus PE, Rymer WZ, Zhou P. Machine learning for supporting diagnosis of amyotrophic lateral sclerosis using surface electromyogram. IEEE Trans Neural Syst Rehabilit Eng. 2014;22(1):96-103. https://doi.org/10.1109/ TNSRE.2013.2274658.

41. Hazarika A, Dutta L, Barthakur M, Bhuyan M. A multiview discriminant feature fusion-based nonlinear process assessment and diagnosis: application to medical diagnosis. IEEE Trans Instrum Meas. 2019;68(7):2498-506. https://doi. org/10.1109/TIM.2018.2866744.

42. Gokgoz E, Subasi A. Effect of multiscale PCA de-noising on EMG signal classification for diagnosis of neuromuscular disorders. J Med Syst. 2014;38(4):31.

43. Ambikapathy B, Kirshnamurthy K, Venkatesan R. Assessment of electromyograms using genetic algorithm and artificial neural networks. Evolut Intell. 2018;14:1-11.

44. Doulah ABMSU, Fattah SA, Zhu WP, Ahmad MO. Wavelet domain feature extraction scheme based on dominant motor unit action potential of EMG signal for neuromuscular disease classification. IEEE Trans Biomed Circuits Syst. 2014;8(2):155-64. https://doi.org/10.1109/TBCAS.2014.2309252.

45. Vallejo M, Gallego CJ, Duque-Muñoz L, Delgado-Trejos E. Neuromuscular disease detection by neural networks and fuzzy entropy on time-frequency analysis of electromyography signals. Expert Syst. 2018;35(4):12274

46. Gokgoz E, Subasi A. Comparison of decision tree algorithms for EMG signal classification using DWT. Biomed Signal Process Control. 2015;18:138-44. 
47. Xia Y, Gao Q, Ye Q. Classification of gait rhythm signals between patients with neuro-degenerative diseases and normal subjects: experiments with statistical features and different classification models. Biomed Signal Process Control. 2015;18:254-62. https://doi.org/10.1016/j.bspc.2015.02.002.

48. Ren P, Tang S, Fang F, Luo L, Xu L, Bringas-Vega ML, Yao D, Kendrick KM, Valdes-Sosa PA. Gait rhythm fluctuation analysis for neurodegenerative diseases by empirical mode decomposition. IEEE Trans Biomed Eng. 2017;64(1):5260. https://doi.org/10.1109/TBME.2016.2536438.

49. Khorasani A, Daliri MR, Pooyan M. Recognition of amyotrophic lateral sclerosis disease using factorial hidden Markov model. Biomed Eng. 2016;61(1):119-26.

50. Welsh R, Jelsone-Swain L, Foerster B. The utility of independent component analysis and machine learning in the identification of the amyotrophic lateral sclerosis diseased brain. Front Hum Neurosci. 2013;7:251. https://doi.org/10 3389/fnhum.2013.00251.

51. Ferraro PM, Agosta F, Riva N, Copetti M, Spinelli EG, Falzone Y, Sorarù G, Comi G, Chiò A, Filippi M. Multimodal structural MRI in the diagnosis of motor neuron diseases. Neurolmage Clin. 2017;16:240-7.

52. Sorbello R, Tramonte S, Giardina ME, La Bella V, Spataro R, Allison B, Guger C, Chella A. A human-humanoid interaction through the use of BCI for locked-in ALS patients using neuro-biological feedback fusion. IEEE Trans Neural Syst Rehabilit Eng. 2018;26(2):487-97.

53. Liu Y-H, Huang S, Huang Y-D. Motor imagery EEG classification for patients with amyotrophic lateral sclerosis using fractal dimension and fisher's criterion-based channel selection. Sensors. 2017;17(7):1557.

54. Mainsah BO, Collins LM, Colwell KA, Sellers EW, Ryan DB, Caves K, Throckmorton CS. Increasing BCI communication rates with dynamic stopping towards more practical use: an ALS study. J Neural Eng. 2015;12(1):016013. https://doi. org/10.1088/1741-2560/12/1/016013.

55. Miao Y, Yin E, Allison BZ, Zhang Y, Chen Y, Dong Y, Wang X, Hu D, Chchocki A, Jin J. An ERP-based BCI with peripheral stimuli: validation with ALS patients. Cogn Neurodyn. 2020;14(1):21-33.

56. van der Burgh HK, Schmidt R, Westeneng H-J, de Reus MA, van den Berg LH, van den Heuvel MP. Deep learning predictions of survival based on MRI in amyotrophic lateral sclerosis. Neurolmage Clin. 2017;13:361-9.

57. Phinyomark A, Phukpattaranont P, Limsakul C. Feature reduction and selection for EMG signal classification. Expert Syst Appl. 2012;39(8):7420-31. https://doi.org/10.1016/j.eswa.2012.01.102.

58. Graimann B, Allison B, Pfurtscheller G. Brain-computer interfaces: a gentle introduction. In: Brain-computer interfaces. New York: Springer; 2009. p. 1-27.

59. Herff C, Heger D, de Pesters A, Telaar D, Brunner P, Schalk G, Schultz T. Brain-to-text: decoding spoken phrases from phone representations in the brain. Front Neurosci. 2015;9:217. https://doi.org/10.3389/fnins.2015.00217.

60. Anumanchipalli GK, Chartier J, Chang EF. Speech synthesis from neural decoding of spoken sentences. Nature. 2019;568(7753):493-8. https://doi.org/10.1038/s41586-019-1119-1.

61. Cooney C, Folli R, Coyle D. Optimizing layers improves CNN generalization and transfer learning for imagined speech decoding from EEG. In: 2019 IEEE international conference on systems, man and cybernetics (SMC). 2019. p. 1311-6. https://doi.org/10.1109/SMC.2019.8914246.

62. Dash D, Ferrari P, Wang J. Decoding imagined and spoken phrases from non-invasive neural (meg) signals. Front Neurosci. 2020;14:290. https://doi.org/10.3389/fnins.2020.00290

63. Dash D, Ferrari P, Hernandez A, Heitzman D, Austin SG, Wang J. Neural speech decoding for amyotrophic lateral sclerosis. Proc Interspeech. 2020;2020:2782-6. https://doi.org/10.21437/Interspeech.2020-3071.

64. Tamura H, Yan M, Sakurai K, Tanno K. EOG-sEMG human interface for communication. Intell Neurosci. 2016;2016:15. https://doi.org/10.1155/2016/7354082.

65. Pinheiro CG, Naves EL, Pino P, Losson E, Andrade AO, Bourhis G. Alternative communication systems for people with severe motor disabilities: a survey. BioMed Eng OnLine. 2011;10(1):31. https://doi.org/10.1186/1475-925X-10-31.

66. Hori J, Sakano K, Saitoh Y. Development of communication supporting device controlled by eye movements and voluntary eye blink. In: The 26th annual international conference of the IEEE engineering in medicine and biology society, vol. 2. 2004. p. 4302-5.

67. Fathi A, Abdali-Mohammadi F. Camera-based eye blinks pattern detection for intelligent mouse. Signal Image Video Process. 2015;9(8):1907-16. https://doi.org/10.1007/s11760-014-0680-1.

68. Harezlak K, Kasprowski P. Application of eye tracking in medicine: a survey, research issues and challenges. Comput Med Imaging Graph. 2018;65:176-90. https://doi.org/10.1016/j.compmedimag.2017.04.006.

69. Villanueva A, Daunys G, Hansen DW, Böhme M, Cabeza R, Meyer A, Barth E. A geometric approach to remote eye tracking. Univ Access Inf Soc. 2009;8(4):241. https://doi.org/10.1007/s10209-009-0149-0.

70. Zhao Q, Yuan X, Tu D, Lu J. Eye moving behaviors identification for gaze tracking interaction. J Multimodal User Interfaces. 2015:9(2):89-104. https://doi.org/10.1007/s12193-014-0171-2.

71. Liu Y, Lee B-S, Rajan D, Sluzek A, McKeown MJ. CamType: assistive text entry using gaze with an off-the-shelf webcam. Mach Vis Appl. 2019;30(3):407-21. https://doi.org/10.1007/s00138-018-00997-4.

72. Wang J, Kothalkar PV, Kim M, Bandini A, Cao B, Yunusova Y, Campbell TF, Heitzman D, Green JR. Automatic prediction of intelligible speaking rate for individuals with ALS from speech acoustic and articulatory samples. Int I Speech Lang Pathol. 2018;20(6):669-79. https://doi.org/10.1080/17549507.2018.1508499.

73. Wisler A, Teplansky K, Green J, Yunusova Y, Campbell T, Heitzman D, Wang J. Speech-based estimation of bulbar regression in amyotrophic lateral sclerosis. In: Proceedings of the eighth workshop on speech and language processing for assistive technologies. Association for Computational Linguistics, Minneapolis, Minnesota; 2019. p. 24-31. https://doi.org/10.18653/v1/W19-1704. https://www.aclweb.org/anthology/W19-1704.

74. Cedarbaum JM, Stambler N, Malta E, Fuller C, Hilt D, Thurmond B, Nakanishi A. The ALSFRS-R: a revised ALS functional rating scale that incorporates assessments of respiratory function. J Neurol Sci. 1999;169(1):13-21. https://doi. org/10.1016/S0022-510X(99)00210-5.

75. Schuster C, Hardiman O, Bede P. Development of an automated MRI-based diagnostic protocol for amyotrophic lateral sclerosis using disease-specific pathognomonic features: a quantitative disease-state classification study. PLoS ONE. 2016;11(12):e0167331. 
76. Wang J, Kothalkar PV, Cao B, Heitzman D. Towards automatic detection of amyotrophic lateral sclerosis from speech acoustic and articulatory samples. Interspeech. 2016;2016:1195-9. https://doi.org/10.21437/Interspeech.2016-1542.

77. Suhas B, Mallela J, Illa A, Yamini B, Atchayaram N, Yadav R, Gope D, Ghosh PK. Speech task based automatic classification of ALS and Parkinson's disease and their severity using log Mel spectrograms. In: 2020 international conference on signal processing and communications (SPCOM). 2020. p. 1-5. https://doi.org/10.1109/SPCOM50965.2020.91795 03 .

78. An K, Kim M, Teplansky K, Green J, Campbell T, Yunusova Y, Heitzman D, Wang J. Automatic early detection of amyotrophic lateral sclerosis from intelligible speech using convolutional neural networks. Proc Interspeech. 2018;2018:1913-7. https://doi.org/10.21437/Interspeech.2018-2496.

79. Vieira H, Costa N, Sousa T, Reis S, Coelho L. Voice-based classification of amyotrophic lateral sclerosis: where are we and where are we going? a systematic review. Neurodegener Dis. 2019;19(5-6):163-70. https://doi.org/10.1159/ 000506259.

80. Wisler A, Teplansky K, Heitzman D, Wang J. The effects of symptom onset location on automatic amyotrophic lateral sclerosis detection using the correlation structure of articulatory movements. J Speech Lang Hear Res. 2021. https:// doi.org/10.1044/2020_JSLHR-20-00288.

81. Stegmann GM, Hahn S, Liss J, Shefner J, Rutkove S, Shelton K, Duncan CJ, Berisha V. Early detection and tracking of bulbar changes in ALS via frequent and remote speech analysis. NPJ Digit Med. 2020;3:132. https://doi.org/10.1038/ s41746-020-00335-x.

\section{Publisher's Note}

Springer Nature remains neutral with regard to jurisdictional claims in published maps and institutional affiliations.

- fast, convenient online submission

- thorough peer review by experienced researchers in your field

- rapid publication on acceptance

- support for research data, including large and complex data types

- gold Open Access which fosters wider collaboration and increased citations

- maximum visibility for your research: over 100M website views per year

At BMC, research is always in progress.

Learn more biomedcentral.com/submissions 